gag or cork should be placed between the back teeth on one side and secured by an attached silk thread wound round the pinna of the same side. Few of those afflicted relish this cork cure, preferring the sympathy excited by their complaint and the gastric comfortor discomfort-of air-gulping. Preventive treatment is simple: if the trick and habit are explained to the patient, and advice is given as to correct and regular feeding, no meal being missed so as to create an empty stomach, this may then be all that is required. It will be well, however, to look carefully for gall-bladder mischief, which, if found, should be corrected.

\section{Dilatation of Stomach}

In a few cases it seems pretty certain that the habit of air-swallowing is learned during convalescence in bed after. confinement or illness or operation. A fluid diet is usual at this time, swallowed in gulps while recumbent. In this way the habit may be quickly discovered by the neurotic. However, not all "aerophagists" are neurotic. In a class apart are those patients after laparotomy-at any age, male or female-who, because of nausea, extreme thirst, or dry tongue and mouth, repeatedly go through the act of swallowing saliva and air together so as to obtain some relief. Thirst is assuaged by the nurse giving to the supine patient sips of water out of a teaspoon or mug, or pills of ice to suck, so encouraging chance air-swallowing. Much air may thus find its way into the silent, perhaps passive, stomach, which then becomes distended, being unable, because atonic, to empty itself by the act of eructation or else by peristalsis driving the atmospheric air on through the pylorus.

Where such a state of affairs occurs an acute dilatation of the stomach may ensue. Indeed, I am convinced that such is the dire consequence of the unwitting swallowing of atmospheric air which cannot be given vent to readily by the patient because recumbent. This being so, it may not be advisable as a first effort to attempt to recover gastric tonus by the hypodermic use of such substances as pituitary extract, or acetylcholine, eserine, or doryl, until an attempt is made to empty the stomach of air by means of a retained Lyon tube, which, being passed, will evacuate what air is in the stomach as well as prevent further air entering. At the same time the patient should be placed in the Fowler position and over on to the left side so as to give vent to air pocketed in the cardiac end of the stomach.

Conclusions as to aerophagy conclude nothing. It is merely a symptom-an effect rather than a cause; one type, embracing the majority of cases, being harmless, the other harmful-maybe desperately so-in the result; these two types of similar mechanism of productionnamely, in the first case by air-gulping into an actively contractile stomach possessing the power to expel swallowed air upwards by way of the oesophagus or downwards through the intermittingly relaxed pyloric sphincter; and in the other case by air-swallowing into a passive, dangerously atonic stomach-often leads on to a fatal issue by an uncontrolled acute gastric dilatation.

Intra-laryngo-tracheal Goitre.-Different views as to the causation of goitre appearing in the submucous tissues of the larynx and trachea are reviewed, with the relevant evidence from histological examination of human and rat (experimental) goitres. A connexion with the ordinary extra-laryngeal thyroid tissue is usual.-Pathogenesis of Intra-laryngo-tracheal Goitre. C. Wegelin.-Schweiz. med. Wschr., July 1, 1939, 69, 593.

\section{THE WEAK “A" REACTION FOUND IN SOME CASES OF THE BLOOD GROUP “AB"}

\author{
BY \\ G. L. TAYLOR, M.D., Ph.D. \\ R. R. RACE, M.R.C.S., L.R.C.P. \\ AILEEN M. PRIOR, M.Sc. \\ AND
}

ELIZABETH W. IKIN, B.Sc.

(The Galton Laboratory Serum Unit. From the Galton Laboratory, University College, London, at the Department of Pathology, University of Cambridge)

It is well known that the reactions of the agglutinogen $A$ with anti-A serum are often markedly weaker when that antigen occurs with $B$ in Group $A B$ than when it occurs alone in Group A. Thomsen (1929) and Thomsen, Friedenreich, and Worsaae (1930) have shown that in some $A B$ cases the factor $B$ is partially dominant to and obscures the $A$ antigen. While there tends to be some weakening of the $B$ reaction in Group $A B$, it is nothing like so marked or so important as is the suppression of $\mathbf{A}$. As there are two types of $A$ antigen $-A_{1}$ and $A_{2}$, a stronger and $a$ weaker - the suppression of $B$ by an already weak $A_{2}$ in the Group $A_{2} B$ may result in it only being possible to detect the $A$ factor with powerful anti-A serum; weaker sera of only moderate strength will often fail completely to reveal this $\mathrm{A}$ factor, and the cells will be diagnosed as belonging to Group B. Cases diagnosed as $B$ have been found on re-examination to belong to Group $A B$, and an inspection of published figures from all parts of the world shows that in a large proportion of the series there is a real deficiency of the numbers in Group AB.

In the accompanying table are given in full the results of titrating against standard cells of the types $\mathbf{A}_{1}, \mathbf{A}_{2}$, $A_{1} B$, and $A_{2} B$, consecutive anti- $A(a)$ sera from fifteen persons, bled because their sera had been found to contain the antibody in amount sufficient to satisfy the minimum requirements of this Unit-a titre of at least 64 with standard $\mathbf{A}_{1}$ cells. The technique used for these titrations has been described by Taylor and Ikin (1939). Although we feel that the use of an anti-A serum with a titre of 64 would be justified if a stronger one were not available, we prefer a higher titre; and, while anti-A sera with titres of 64 are being stored in case they are needed at a time when the demand may be very great, stronger ones are being sent to those who apply to us for grouping serum.

Of the sera from 378 persons of Group B, $111(29.37$ per cent.) had titres of at least 64 . Only 31 ( 8.2 per cent.) had titres of 128 or more, and, as Group B forms about 9 per cent. of the English population, it has been necessary to explore some 4,200 people to get thirty-one adequate sera-that is, only 0.74 per cent. of the population provided really good anti-A serum.

As the factor $B$ does not occur in strong and weak forms, anti-B $(\beta)$ serum with a titre of at least 64 with standard B cells seems to be adequate; 1,347 persons of Group A yielded sixty-seven satisfactory sera-that is, 4.97 per cent. of all A's, or 2.19 per cent. of the whole population, assuming that Group $A$ is 44 per cent. thereof.

Apart from the $A_{1} B$ cells used with serum 13 the four donors of the control cells were always the same persons, and the table shows that the order of decreasing strength of reaction is $A_{1}, A_{1} B, A_{2}, A_{2} B$. This same gradation in strength, $A_{1}, A_{1} B, A_{2}, A_{2} B$, was also evident in the comparative reactions given by cells from large numbers of 
Results of Titrating against Standard Cells Consecutive Anti-A (a) Sera from Fifteen Persons

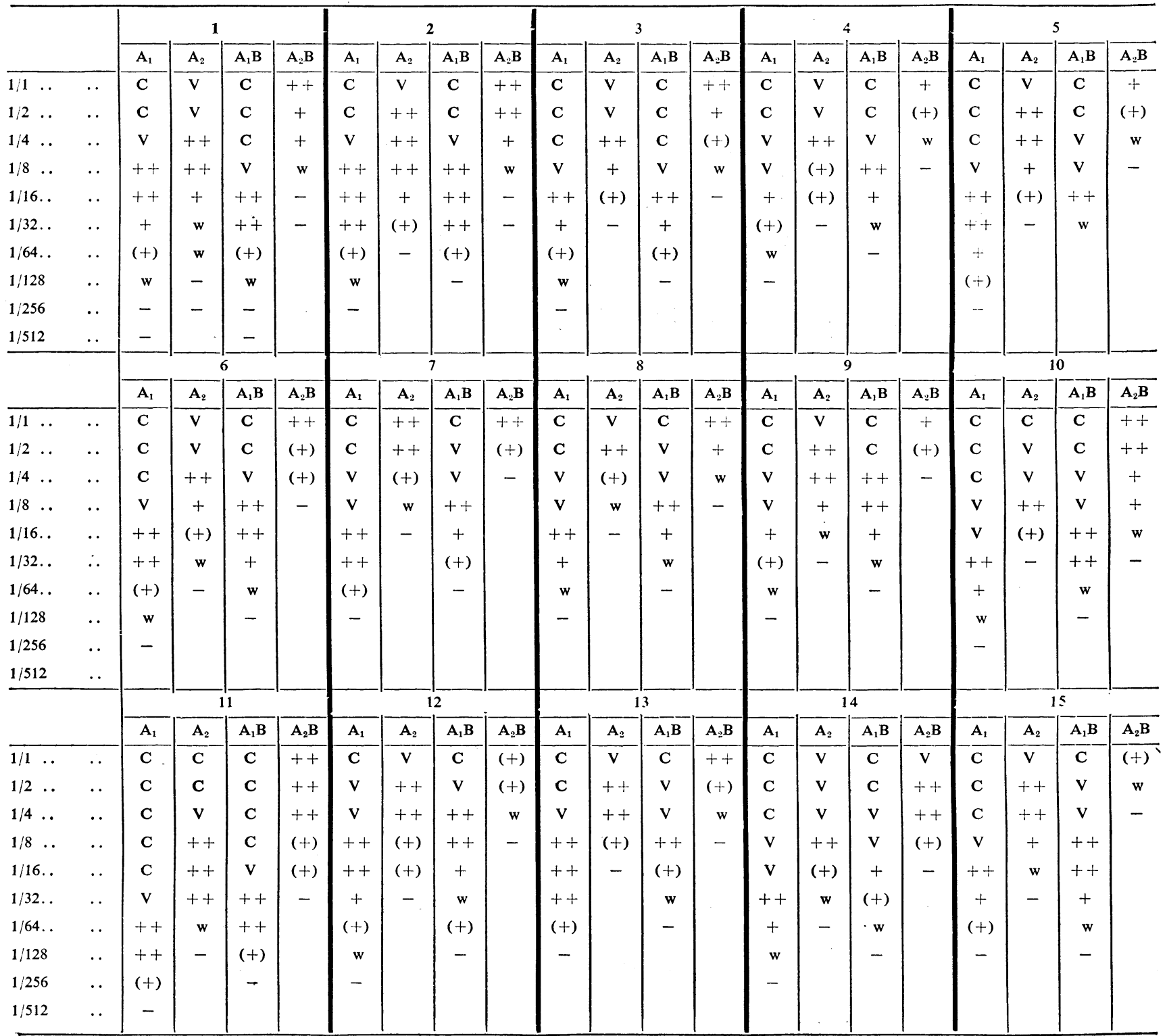

No reaction is scored as negative without a microscopical examination; reactions weaker than $\mathrm{V}$ were determined with a microscope. $\mathrm{C}=$ clumps of cells in clear surrounding fluid visible to naked eye

$\mathrm{V}=$ somewhat weaker reaction, with clumps distinctly visible to naked eye, although surrounding fluid is not clear.

$++=$ very big clumps under microscope $;+=$ not quite such big clumps $;(+)=$ less than + other donors examined. With none of the fifteen sera
was the $\mathrm{A}_{2} \mathrm{~B}$ reaction very big, and with many it was very small indeed. With each serum tests were also made by the slide metbod, and while a reaction with $\mathrm{A}_{2} \mathrm{~B}$ cells was always found, it was sometimes very trifling and delayed, and difficult to see with the naked eye. It was quite evident that even with sera that might well be considered to be of good strength the greatest care is necessary if some $A B$ cases are not to be diagnosed as $B$.

Even the examination of the serum for antibodies would not necessarily disclose that a case diagnosed as $\mathbf{B}$ was really $A B$, for the serum from quite a number of $A_{2} B$ cases contains the antibody $\alpha_{1}$, which reacts with $A_{1}$ but not with $A_{2}$ cells, and if this serum were mixed with test-cells of the type $A_{1}$ a reaction would result and seem to confirm the diagnosis of Group B. As the antibody $\alpha_{1}$ does not react with $A_{2}$ cells the testing of the serum of all apparent $B$ 's with $A_{2}$ cells should prevent an $A_{2} B$ being mistaken for a $\mathrm{B}$; for if a person's serum contains the antibody $\alpha$, which reacts with both $A_{1}$ and $A_{2}$ cells, that person's cells cannot be either $A_{1} B$ or $A_{2} B{ }^{*}$ The sera of all $B$ persons examined by this Unit are tested with $\mathbf{A}_{2}$ cells.

Very occasionally $A B$ cells are diagnosed as $A$; this mistake ought never to occur, and can only be due to the use of an anti-B serum which is far too weak.

\section{Summary}

Copies of protocols are given which illustrate how weakly some AB cells react with anti-A serum, and it is shown that certain persons of Gröup $\mathrm{AB}$ may easily be diagnosed as $\mathrm{B}$.

\section{REFERENCES}

Taylor, G. L., and Ikin, Elizabeth W. (1939). British Medical Journal, 1, 1027. Fr. (1929). Z. Rassenphy'siol., 1, 198. - Friedenreich, V., and Worsaae, E. (1930). Ibid., 3. 20.

* This test would fail to disclose the $A$ factor in the unlikely case of an $A_{1} B$ person, misdiagnosed as $B$, whose serum contained the rare agglutinin $a_{2}$ (anti-O). Testing all reputed B's with both $\mathrm{O}$ and $\mathrm{A}_{2}$ cells would eliminate this source of error, and, of course, there would be no reaction with $A_{1}$ cells. 